\title{
Interpretable Monotonicities for Entropies of Intuitionistic Fuzzy Sets or Interval-valued Fuzzy Sets
}

\author{
Christophe Marsala ${ }^{a}$ and Bernadette Bouchon-Meunier ${ }^{b}$ \\ ${ }^{a}$ Sorbonne Université, CNRS, LIP6, F-75005 Paris, France, Christophe.Marsala@lip6.fr \\ ${ }^{b}$ Sorbonne Université, CNRS, LIP6, F-75005 Paris, France, Address, Bernadette.Bouchon-Meunier@lip6.fr
}

\begin{abstract}
Monotonicity is a major property of entropy measures. In this paper, we focus on entropy measures for intuitionistic fuzzy sets and interval-valued fuzzy sets. We consider their polar representation which provides an easy interpretation of monotonicity for several entropy measures introduced in the literature. Moreover, this polar representation enables us to propose two new kinds of monotonicity for such entropies, that offer a more understandable way to explain differences of entropies. Classical measures of entropy for intuitionistic fuzzy sets are compared with regard to these new forms of monotonicity.
\end{abstract}

Keywords: Entropy, Information measure, Intuionistic fuzzy sets, Interval-valued fuzzy sets, Monotonicity, Polar representation.

\section{Introduction}

Monotonicity is a major property of entropy measures (or entropies for short) [3] that relies on a given order. It may be a partial order, on the sets on which it applies. In the literature, it does not exist a unique definition of a monotonicity property for an entropy measure.

In the fuzzy setting, and in particular, when considering Atanassov Intuitionistic Fuzzy Sets (IFS) [2] or Interval-Valued Fuzzy Sets (IVFS) [23], several entropies have been proposed, often defined from a specific definition of monotonicity $[5,6,7,19]$. Indeed, these two models of representation, IFS and IVFS, are equivalent from a theoretical point of view, even though they are different in practice $[6,8]$. Therefore, entropies for one of this kind of fuzzy sets are usable for the other kind. Such entropies are very useful to measure the information brought out by an IFS or an
IVFS in several kinds of application, such as in decision support systems [22] or in machine learning [4] to cite a few examples.

In order to enable a more interpretable study of entropies in the IFS/IVFS setting, we introduced a polar representation of these kinds of fuzzy sets $[13,15]$. Indeed, it is very promising to manipulate and to study such bipolar information represented as polar coordinates $[13,15,20]$ and we can highlight the gain in interpretability it allows.

In this paper, we propose two definitions of monotonicity in this context. The polar representation allows the introduction of a monotonicity based on the radius and another one based on the angle. They prove to be more easily interpretable as they take into account the two components of the IFS in a global way and represent a measure of the disorder in the values. They are closer to the classic interpretation of entropy than the existing definitions.

The paper is composed as follows. In Section 2, a recall on IFS and IVFS is done and we remind the connections between these two representations. In Section 3, the polar representation of IFS is introduced and the meaning of each of the two components of this representation are highlighted. In Section 4, after a quick recall of existing monotonicity properties and of the three main entropies of IFS, we introduce two new definitions of monotonicity for an entropy of IFS. Section 5 concludes this paper and highlights some future works.

\section{Intuitionistic and Interval-valued Fuzzy Sets}

Let $U=\left\{u_{1}, \ldots, u_{n}\right\}$ be a discrete universe and let $A$ be a subset of $U$ that might be imprecisely defined. The intuitionistic fuzzy sets and the interval-valued fuzzy sets are two representations of the imprecision that point out two different ways of representing the same 
knowledge about the membership of an element $u \in U$ to the subset $A$.

As it has already been shown in the literature, these two models of representation are theoretically equivalent. However, each of them brings out a particular approach to the definition of $A$ and is adapted to specific applications.

\subsection{Intuitionistic Fuzzy Sets (IFS)}

In the fuzzy set community, the intuitionistic fuzzy sets have been introduced by Atanassov [2]. An IFS $A$ of $U$ is defined as:

$$
A=\left\{\left(u, \mu_{A}(u), v_{A}(u)\right) \mid u \in U\right\}
$$

with $\mu_{A}: U \rightarrow[0,1]$ and $v_{A}: U \rightarrow[0,1]$ such that for all $u \in U, 0 \leq \mu_{A}(u)+v_{A}(u) \leq 1$.

The values $\mu_{A}(u)$ and $v_{A}(u)$ are, respectively, the membership degree and the non-membership degree of $u$ to $A$.

The complement $A^{c}$ of the IFS $A$ is defined as the subset $A^{c}=\left\{\left(u, v_{A}(u), \mu_{A}(u)\right) \mid u \in U\right\}$

Two particular cases are the following: $U$ is the IFS such that $\mu_{U}(u)=1$ and $v_{U}(u)=0$ for all $u \in U$, and the empty set is the IFS such that $\mu_{U}(u)=0$ and $v_{U}(u)=1$ for all $u \in U$.

The margin of hesitancy of the membership of $u$ to $A$ is defined as $\pi_{A}(u)=1-\left(\mu_{A}(u)+v_{A}(u)\right)$, it is also called intuitionistic index of $u$ to $A$. This index informs us about the lack of knowledge about $A$ when it exists.

When the margin of hesitancy according to $A$ is equal to zero for all $u$, that is to say when $\mu_{A}(u)+v_{A}(u)=1$, $\forall u \in U$, then $A$ is a Zadeh's fuzzy set.

An IFS $A$ such that $\mu_{A}(u)=v_{A}(u)=0, \forall u \in U$ is said completely intuitionistic [5]. It corresponds to an IFS with a maximal margin of hesitancy, stating that there is no information available concerning the membership or the non-membership to $A$ for elements from $U$.

\subsection{Interval-Valued Fuzzy Sets (IVFS)}

Interval-valued fuzzy sets have been introduced by L. A. Zadeh in 1975 [23]. In the sequel, we use notations classically used in the literature [7, 8].

An IVFS $A$ of $U$ is defined by a function $F_{A}$ from $U$ to $\mathbb{I}([0,1])$, the set of all closed intervals of $[0,1]$, such that for every $u \in U, F_{A}(u)=\left[\underline{f}_{A}(u), \bar{f}_{A}(u)\right]$ with $\underline{f}_{A}(u) \leq \bar{f}_{A}(u)$. The IVFS is then the set $A=$ $\left\{\left(u,\left[\bar{f}_{A}(u), \bar{f}_{A}(u)\right]\right) \mid u \in U\right\}$.

This interval represents a framing of the degree of membership of $u$ to $A$. $f_{A}(u)$ represents the minimum value that can be given to the degree of membership of $u$ to $A$ and $\bar{f}_{A}(u)$ the maximum value that can be given to this degree. The bigger this interval, the less knowledge or reliable information we have on the belonging of $u$ to $A$

When $\underline{f}_{A}(u)=\bar{f}_{A}(u)$ for all $u \in U$, then $A$ is a Zadeh's fuzzy set.

\subsection{IFS or IVFS?}

Various authors have shown that the intuitionistic and interval-based knowledge representations are equivalent. There exists a one-to-one mapping between the set of IFS and the set of IVFS of $U[1,5,7,8]$. In other words, the IFS $A=\left\{\left(u, \mu_{A}(u), v_{A}(u)\right) \mid u \in U\right\}$ can be represented as the IVFS

$$
\left\{\left(u,\left[\mu_{A}(u), 1-v_{A}(u)\right]\right) \mid u \in U\right\} .
$$

Conversely, the IVFS $A=\left\{\left(u,\left[\underline{f}_{A}(u), \bar{f}_{A}(u)\right]\right) \mid u \in U\right\}$ can be represented as the IFS

$$
\left\{\left(u, \underline{f}_{A}(u), 1-\bar{f}_{A}(u)\right) \mid u \in U\right\} .
$$

Figure 1 illustrates the representation of an IVFS and the equivalent IFS.

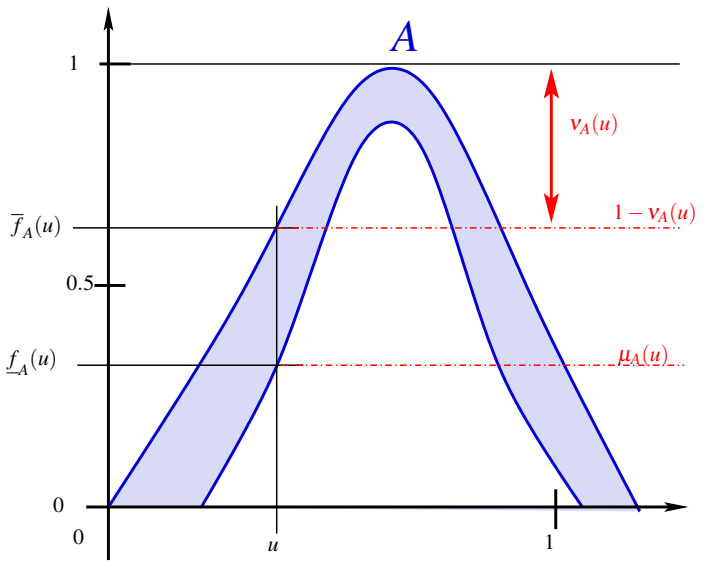

Figure 1: Links between IFS and IVFS.

Thus, it can be concluded that IFS and IVFS are two different representations of a similar phenomenon of the knowledge related to both the membership and the non-membership of an element to a set. As a consequence, the choice of one of these representations depends only of the context of use: one could be more appropriate in a given context than the other. One could conclude that theoretical study related to IFS could easily be adapted to IVFS and reciprocally.

In the following, we have decided to focus on the IFS representation and we argue that the study can also be adapted to IVFS. 


\section{Polar representation of IFS}

An IFS $A=\left\{\left(u, \mu_{A}(u), v_{A}(u)\right) \mid u \in U\right\}$ can be represented in a cartesian space by the set of points $\left(\mu_{A}(u), v_{A}(u)\right)$ defined in $[0,1] \times[0,1]$, for every element $u \in U[13,14,15]$.

It is also possible to use a polar representation for this set of points. Each element $u$ is then represented by $\left(r_{A}(u), \theta_{A}(u)\right)$, where $r_{A}(u)$ is its radius and $\theta_{A}(u)$ its polar angle. The radius is defined by $r_{A}(u)=$ $\sqrt{\mu_{A}(u)^{2}+v_{A}(u)^{2}}$, and it corresponds to the distance between the point and the origin.

The polar angle $\theta_{A}(u)$, such that $\tan \theta_{A}(u)=\frac{v_{A}(u)}{\mu_{A}(u)}$ if $\mu_{A}(u) \neq 0$ and $\theta_{A}(u)=\frac{\pi}{2}$ otherwise, is the angle between the abscissa axis and the line between points $(0,0)$ and $\left(\mu_{A}(u), v_{A}(u)\right)$ (see Figure 2).

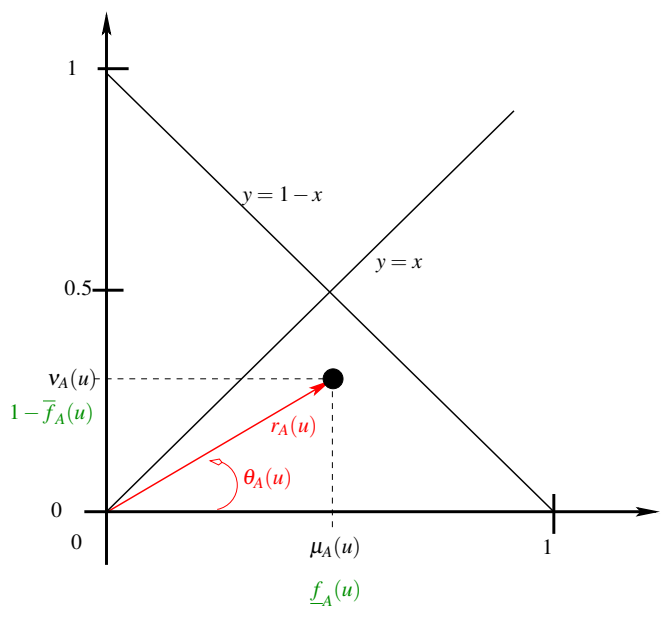

Figure 2: Representations of IFS and IVFS.

In the sequel, to simplify the notations, we denote $r_{A}(u)$ and $\theta_{A}(u)$ by $r$ and $\theta$ when there is no ambiguity.

In [15], this polar representation of IFS allows to study the variations of measures of entropy of IFS and it brings a better interpretation of these variations.

From Section 2.3, we see that it is possible to represent an IVFS in a similar way, as $A=$ $\left\{\left(u,\left[f_{A}(u), \bar{f}_{A}(u)\right]\right) \mid u \in U\right\}$ for the Cartesian coordinates. Obtaining polar coordinates is then done accordingly (Figure 2).

A polar representation of IFS and IVFS is an interesting tool to study them, since radius and polar angle combine both aspects of membership and nonmembership.

\subsection{Semantics for the radius $r$}

Let $\theta$ be given such that $\theta \in\left[0, \frac{\pi}{2}\right]$. In this case, the corresponding radius $r$ varies in $\left[0, \frac{\sqrt{2}}{2 \sin \left(\theta+\frac{\pi}{4}\right)}\right]$. The radius $r$ evaluates the distance from the origin $(0,0)$.

When $r$ is equal to $\frac{\sqrt{2}}{2 \sin \left(\theta+\frac{\pi}{4}\right)}$, it corresponds to the case of a well-known fuzzy set.

For a given $\theta$, the radius $r$ provides an information about the knowledge we have on the membership of $u$ to $A$ : the greater $r$, the smaller the lack of information about the fact that $u$ belongs or not to $A$.

This is close to the information provided by the intuitionistic index $\pi_{A}(u)=1-\left(\mu_{A}(u)+v_{A}(u)\right)$ that could also be rewritten $\pi_{A}(u)=1-r \sqrt{2} \sin \left(\theta+\frac{\pi}{4}\right)$ in a polar representation as given in [15].

\subsection{Semantics for the polar angle $\theta$}

Let $r$ be given such that $r \in[0,1]$. In this case, $\theta$ varies ${ }^{1}$ in $\left[0, \frac{\pi}{2}\right]$.

When $A$ is not completely intuitionistic, three particular values for $\theta$ can be highlighted:

- when $\theta=0$, the corresponding element $u$ belongs to the abscissa axis, thus, $v_{A}(u)=0$ and $u$ belongs "at least a little" to $A$;

- when $\theta=\frac{\pi}{2}$, the corresponding element $u$ belongs to the ordinate axis and thus, $\mu_{A}(u)=0$ and $u$ doesn't belong to $A$;

- when $\theta=\frac{\pi}{4}$, the corresponding element $u$ is on the straight line $y=x$ and $\mu_{A}(u)=v_{A}(u)$, and, thus, the corresponding information is not sufficient to decide whether $u$ belongs or not to $A$.

As a consequence, given $r$, the gap $\left|\theta-\frac{\pi}{4}\right|$ provides us with the information concerning the way it can be decided whether the corresponding $u$ belongs or not to $A$ : the more $\theta$ is far from $\frac{\pi}{4}$, the more one of the degrees (membership or non-membership) is dominating.

\section{Entropies and monotonicities}

In the following, several entropies of IFS are presented. As it has been recalled in Section 2.3, the corresponding presentation could be similarly made for IVFS.

An entropy of IFS is a measure $E$, from $2^{U}$ to $[0,1]$ (or possibly $\mathbb{R}^{+}$according to [5] but with the possibility to

\footnotetext{
${ }^{1}$ When $r$ is greater than $\frac{\sqrt{2}}{2}$ the variations of $\theta$ are restricted in order to define an IFS.
} 
normalise it to $[0,1]$ as the maximum is finite) that fulfils a set of properties that could differ according to the authors. Major differences to define such an entropy could be noted between the definition in [5] and those in $[10,21]$ : the first one is based on the difference between $A$ and a fuzzy set, and the second ones is based on the difference between $A$ and a crisp set.

A summary of these definitions can be given:

i) $E(A)=0$ if and only if:

- $A$ is a crisp set $[10,21]$

- $A$ is a fuzzy set [5]

ii) maximum value for $E(A)$ :

- $E(A)=1$ iff $\mu_{A}(u)=v_{A}(u) \forall u \in U[10,21]$

- $E(A)=|U|$ iff $\mu_{A}(u)=v_{A}(u)=0 \forall u \in U$ [5]

iii) $E(A)=E\left(A^{c}\right)$ for all IFS $A[5,10,21]$

iv) monotonicity property of $E$ related to an order on elements of $2^{U}$ (this property differs according to $[5,21,10]$ as explained hereafter).

Property $i v$ ) states that entropy measures should fulfil a monotonicity property according to a given order on the elements of $2^{U}$. It is a main characteristic of entropies as it has been highlighted that all entropies should satisfy a particular kind of monotonicity [3].

\subsection{Order and entropy}

In the state of the art, a main property for an entropy of IFS is related to a given order between elements of $2^{U}$. In [5], this order is said to be based on the definition by [2], and in [10, 21] it is based on the definition of less fuzzy than. We recall these two orders (M1) and (M2) in the following:

(M1) $E(A) \leq E(B)$, if $A$ is less fuzzy than $B$,

ie. if $\mu_{A}(u) \leq \mu_{B}(u)$ and $v_{A}(u) \geq v_{B}(u)$ when $\mu_{B}(u) \leq$ $v_{B}(u), \forall u \in U$, or $\mu_{A}(u) \geq \mu_{B}(u)$ and $v_{A}(u) \leq v_{B}(u)$ when $\mu_{B}(u) \geq v_{B}(u), \forall u \in U$.

(M2) $E(A) \geq E(B)$ if $A \preceq B$

ie. if $\mu_{A}(u) \leq \mu_{B}(u)$ and $v_{A}(u) \leq v_{B}(u), \forall u \in U$,

More generally, any definition of monotonicity could be used to define a particular entropy $E[3,13,15]$.

However, interpreting an order relation could be difficult and non-intuitive. For instance, the impact of the definition of an entropy by means of each of these two order relations is presented in Figure 3 for (M1) and Figure 4 for (M2).

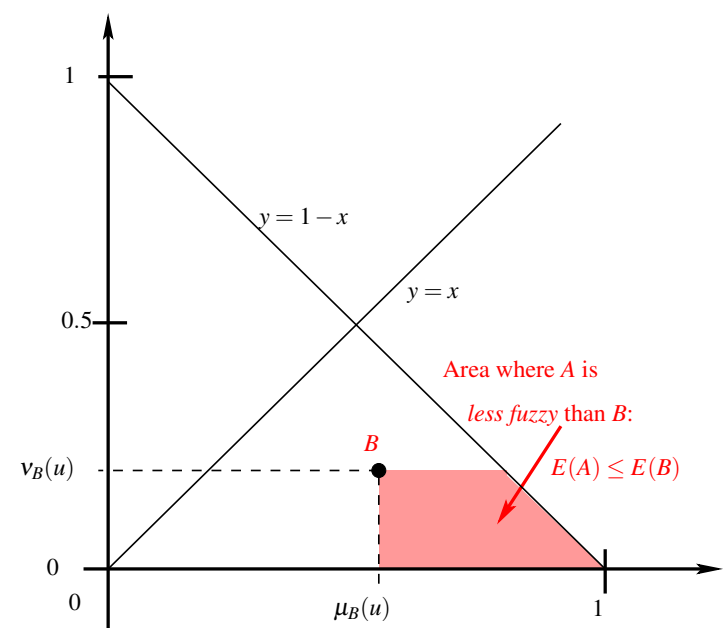

Figure 3: Example for order relation (M1).

These two orders lead to distinct behaviours. Moreover, it should be noted that these orders are not clearly interpretable. For instance, in Figure 3, it is easy to see that there exist some $A$ 's that are completely fuzzy and considered as "less fuzzy than $B$ ".

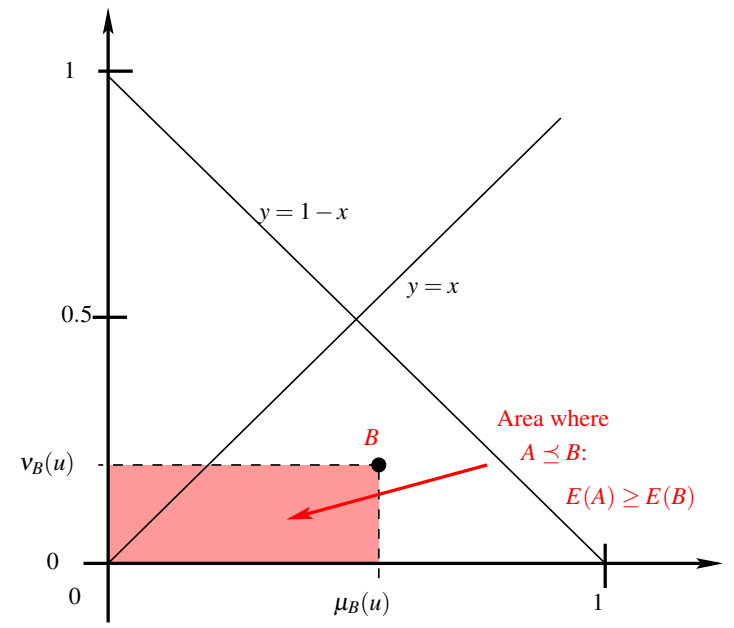

Figure 4: Example for order relation (M2).

\subsection{New definitions of monotonicity}

In this paper, our aim is to propose a more interpretable and intuitive view of the monotonicity properties for the entropy of an IFS in order to provide an interpretation closer to the expectations of the user of such a measure. Two new monotonicity properties are introduced in this sense hereafter.

In the following, let $A$ be an IFS (or, similarly, an IVFS) of $U=\left\{u_{1}, \ldots, u_{n}\right\}$. 


\subsubsection{Radius monotonicity}

As it has been recalled in Section 3.1, for a given $u \in U$, the value of $r_{A}(u)$ brings out some knowledge about the membership or non-membership of $u$ to $A$. The greater $r_{A}(u)$, the more information we have about the membership or non-membership of $u$ to $A$. This can be used to define a partial order on IFS (resp. IVFS): let $A$ and $B$ be two IFS (resp. IVFS) of $U, A$ is said to be better known than $B$ when $r_{A}(u) \geq r_{B}(u)$ for all $u \in U$.

A monotonicity property related to a radius $r$ for a given measure $E$ can thus be defined as:

Definition 4.1 (Radius monotonicity). $E(A) \leq E(B)$ if $A$ is better known than $B$. That is to say: $E(A) \leq E(B)$ if $r_{A}(u) \geq r_{B}(u)$ for all $u \in U$.

This monotonicity is illustrated in Figure 5.

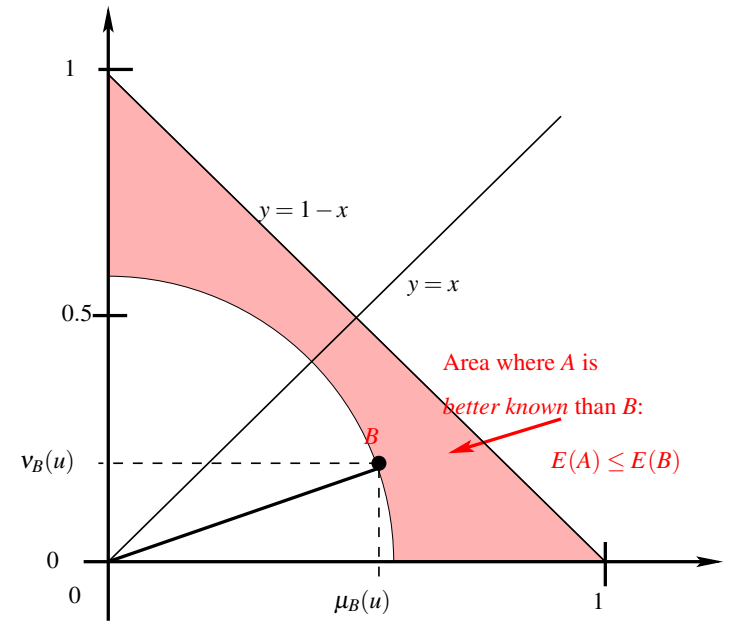

Figure 5: Example for radius Monotonicity.

A radius monotonous entropy measures a disorder and a better knowledge of the studied phenomenon entails a smaller value of such an entropy.

\subsubsection{Polar angle monotonicity}

As it has been shown in Section 3.2, the greater $\left|\theta_{A}(u)-\frac{\pi}{4}\right|$, the easier the decision according to the fact that $u$ belongs or not to $A$. Thus, the value $\left|\theta_{A}(u)-\frac{\pi}{4}\right|$ provides an information about the possibility to decide firmly on the membership of $u$ to $A$. The decision is related here to the question of which side of the (fuzzy) border of $A$ is $u$.

A partial order on IFS (resp. IVFS) can be defined according to this: let $A$ and $B$ be two IFS (resp. IVFS) of $U . A$ is said to be more informative about the decision related to the membership of $u$ than $B$ when $\left|\theta_{A}(u)-\frac{\pi}{4}\right| \geq\left|\theta_{B}(u)-\frac{\pi}{4}\right|$ for all $u \in U$.
Definition 4.2 (Polar angle monotonicity.). $E(A) \leq$ $E(B)$ when $A$ is more informative about the decision than $B$. That is to say: $E(A) \leq E(B)$ when $\left|\theta_{A}(u)-\frac{\pi}{4}\right| \geq\left|\theta_{B}(u)-\frac{\pi}{4}\right|$ for all $u \in U$.

This monotonicity is illustrated in Figure 6.

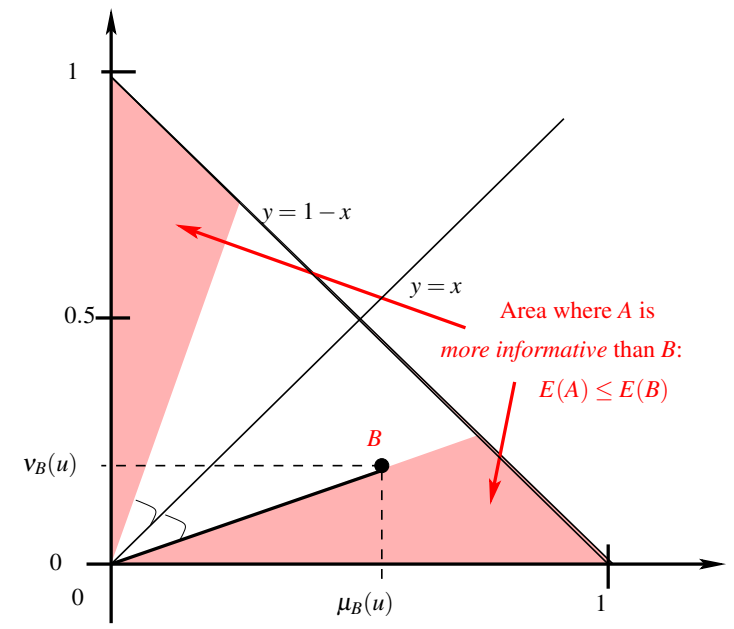

Figure 6: Example for polar angle Monotonicity.

The polar angle monotonicity is associated with the possibility to decide on which side of the set border a given element is: the higher $E(A)$, the more difficult it is to decide on the membership of elements to $A$. A polar-angle monotonous entropy is then useful to evaluate the easiness to make a decision for a given set.

\subsection{Application to entropies of IFS}

In [15], three measures of entropy of IFS have been studied in the polar form and we present them in this section.

In the sequel, to simplify notations, we respectively denote $\mu_{A}\left(u_{i}\right), v_{A}\left(u_{i}\right), r_{A}\left(u_{i}\right)$ and $\theta_{A}\left(u_{i}\right)$ by $\mu_{i}, v_{i}, r_{i}$ and $\theta_{i}$ when there is no ambiguity.

The entropy measure of an IFS $A$ of $U$ introduced by Szmidt and Kacprzyk [21] is written as follows in a polar representation:

$$
E_{1}(A)=1-\frac{\sqrt{2}}{2 n} \sum_{i=1}^{n} r_{i}\left|\sin \left(\frac{\pi}{4}-\theta_{i}\right)\right| .
$$

This measure has a form close to one of the entropies defined by Hung [11] that could thus be handled in the same way.

The entropy measure introduced by Burillo and Bustince [5] is written as:

$$
E_{2}(A)=n-\sqrt{2} \sum_{i=1}^{n} r_{i} \sin \left(\theta_{i}+\frac{\pi}{4}\right) .
$$



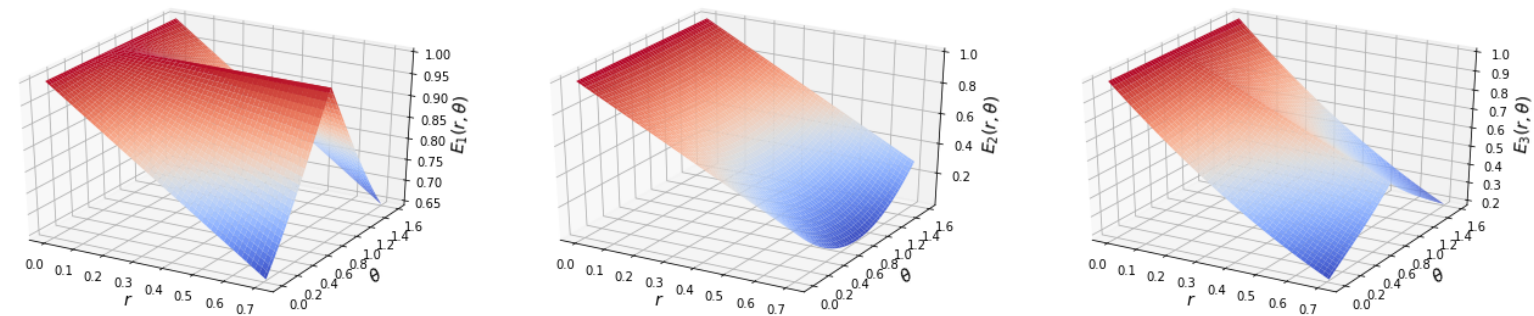

Figure 7: Variations of $E_{1}, E_{2}$ and $E_{3}$

Finally, the measure proposed by Guo and Song [10] is the following:

$$
\begin{array}{r}
E_{3}(A)=\frac{1}{2 n} \sum_{i=1}^{n}\left(1-r_{i} \sqrt{2}\left|\sin \left(\frac{\pi}{4}-\theta_{i}\right)\right|\right) \\
\cdot\left(2-r_{i} \sqrt{2} \sin \left(\frac{\pi}{4}+\theta_{i}\right)\right) .
\end{array}
$$

The variations of $E_{1}, E_{2}$ and $E_{3}$ when $r$ varies in $\left[0, \frac{\sqrt{2}}{2}\right]$ and $\theta$ varies in $\left[0, \frac{\pi}{2}\right]$ are shown in Figure 7 .

\subsubsection{Monotonicity with regard to $r$}

In order to focus on the way the entropies vary in relation with the radius $r$, we consider $U$ reduced to a unique element $u$ and an IFS $A$ defined on $U$. It can be noted that a corresponding study could be done on a set $U$ such that $|U|>1$ and focusing on the variations concerning a single element $u \in U$.

It can be easily verified that the three entropy measures $E_{1}, E_{2}$ and $E_{3}$ are radius monotonous as they satisfy the monotonicity with regard to $r$, as defined in Section 4.2 (an illustration of their variations can be found in [15]).

In fact, it is sufficient to see that the three measures are decreasing in $r$ for a fixed value of $\theta$, which indicates a desirable property for entropies.

\subsubsection{Monotonicity with regard to $\theta$}

Still considering $U$ reduced to a unique element $u$, we can see that the three measures have different behaviours (an illustration of their variations in this case can also be found in [15]).

For a fixed value of $r$, on the one hand the two measures $E_{1}$ and $E_{3}$ are increasing for $\theta \in\left[0, \frac{\pi}{4}\right]$ and decreasing for $\theta \in\left[\frac{\pi}{4}, \frac{\pi}{2}\right]$. On the other hand, the measure $E_{2}$ has an opposite behaviour as it is decreasing for $\theta \in\left[0, \frac{\pi}{4}\right]$ and increasing for $\theta \in\left[\frac{\pi}{4}, \frac{\pi}{2}\right]$. As a consequence, $E_{1}$ and $E_{3}$ are polar-angle monotonous as they fit the definition of polar angle monotonicity. On the contrary, $E_{2}$ is not polar-angle monotonous.

\section{Conclusion and future work}

In this paper, two new definitions of monotonicity for entropy measures of intuitionistic fuzzy sets are introduced. They are based on the polar representation of these sets, with the purpose of improving the interpretability of these entropies. Thus we point out definitions of entropy measures that are semantically closer to the classic definition of entropy.

In future works, these definitions will be used to propose a more general framework for the definition of entropies of IFS and IVFS. Such a study could also be proposed for parametric entropies, such as [12, 18] which are based on the order M1, or for entropies generated by other orders, for instance "sharpened" [9], or defined from divergences $[16,17]$.

\section{References}

[1] K. Atanassov, G. Gargov, Interval valued intuitionistic fuzzy sets, Fuzzy Sets and Systems 31 (3) (1989) 343 - 349.

[2] K. T. Atanassov, Intuitionistic fuzzy sets, Fuzzy Sets and Systems 20 (1986) 87-96.

[3] B. Bouchon-Meunier, C. Marsala, Entropy and monotonicity in artificial intelligence, International Journal of Approximate Reasoning.

[4] P. Bujnowski, E. Szmidt, J. Kacprzyk, Intuitionistic fuzzy decision tree: a new classifier, in: Intelligent Systems' 2014, Springer, 2015, pp. 779790 .

[5] P. Burillo, H. Bustince, Entropy on intuitionistic fuzzy sets and on interval-valued fuzzy sets, Fuzzy Sets and Systems 78 (1996) 305-316.

[6] H. Bustince, E. Barrenechea, M. Pagola, J. Fernandez, Z. Xu, B. Bedregal, J. Montero, H. Hagras, F. Herrera, B. D. Baets, A historical account of types of fuzzy sets and their relationships, IEEE Transactions on Fuzzy Systems 24 (2016) 179-194. 
[7] I. Couso, H. Bustince, From fuzzy sets to interval-valued and Atanassov intuitionistic fuzzy sets: A unified view of different axiomatic measures, IEEE Transactions on Fuzzy Systems 27 (2) (2019) 362-371.

[8] D. Dubois, H. Prade, Interval-valued fuzzy sets, possibility theory and imprecise probability, in: Conference EUSFLAT-LFA'05, Barcelona, Spain, 2005, pp. 314-319.

[9] P. Grzegorzewski, E. Mrowka, On the entropy of intuitionistic fuzzy sets and interval-valued fuzzy sets, in: Proc. of the IPMU 2004, Perugia, Italy, 2004, pp. 1419-1426.

[10] K. Guo, Q. Song, On the entropy for Atanassov's intuitionistic fuzzy sets: an interpretation from the perspective of amount of knowledge, Applied Soft Computing 24 (2014) 328-340.

[11] W.-L. Hung, A note on entropy of intuitionistic fuzzy sets, International Journal of Uncertainty, Fuzziness and Knowledge-Based Systems 11 (05) (2003) 627-633.

[12] R. Joshi, S. Kumar, Parametric (r,s)-norm entropy on intuitionistic fuzzy sets with a new approach in multiple attribute decision making, Fuzzy Information and Engineering 9 (2) (2017) 181-203.

[13] C. Marsala, B. Bouchon-Meunier, Entropies et ensembles flous intuitionistes, in: Rencontres Francophones sur la Logique Floue et ses Applications (LFA'2019), Cépaduès, 2019, pp. 143148.

[14] C. Marsala, B. Bouchon-Meunier, Interprétabilité des entropies d'ensembles flous intuitionnistes ou définis par intervalles, in: Rencontres Francophones sur la Logique Floue et ses Applications (LFA'2020), Cépaduès, 2020, pp. 141-147.

[15] C. Marsala, B. Bouchon-Meunier, Polar representation of bipolar information: A case study to compare intuitionistic entropies, in: M.-J. Lesot, S. Vieira, M. Z. Reformat, J. P. Carvalho, A. Wilbik, B. Bouchon-Meunier, R. R. Yager (Eds.), International Conference on Information Processing and Management of Uncertainty in Knowledge-Based Systems (IPMU'2020), Springer, 2020, pp. 107-116.

[16] I. Montes, S. Montes, N. Pal, On the use of divergences for defining entropies for Atanassov intuitionistic fuzzy sets, in: Advances in Fuzzy Logic and Technology 2017, Springer, 2017, pp. 554565 .
[17] I. Montes, N. Pal, S. Montes, Entropy measures for Atanassov intuitionistic fuzzy sets based on divergence, Soft Computing 22 (2018) 50515071.

[18] S. Singh, S. Sharma, On a generalized entropy and dissimilarity measure in intuitionistic fuzzy environment with applications, Soft Computing (25) (2021) 7493-7514.

[19] V. Singh, S. P. Yadav, R. Mesiar, Information measures in atanassov's intuitionistic fuzzy environment and their application in decision making, IEEE Transactions on Fuzzy Systems 28 (11) (2019) 2905-2917.

[20] L. H. Son, R. T. Ngan, M. Ali, H. Fujita, M. Abdel-Basset, N. L. Giang, G. Manogaran, M. K. Priyan, A new representation of intuitionistic fuzzy systems and their applications in critical decision making, IEEE Intelligent Systems 35 (1) (2020) 6-17.

[21] E. Szmidt, J. Kacprzyk, New measures of entropy for intuitionistic fuzzy sets, in: Proceedings of the Ninth Int. Conf. on Intuitionistic Fuzzy Sets (NIFS), Vol. 11, Sofia, Bulgaria, 2005, pp. 12-20.

[22] E. Szmidt, J. Kacprzyk, P. Bujnowski, How to measure the amount of knowledge conveyed by atanassov's intuitionistic fuzzy sets, Information Sciences 257 (2014) 276-285.

[23] L. Zadeh, The concept of a linguistic variable and its application to approximate reasoning, part 1, Information Sciences 8 (1975) 199-249, reprinted in "Fuzzy Sets and Applications: selected papers by L. A. Zadeh", R. R. Yager, S. Ovchinnikov, R. M. Tong and H. T. Nguyen eds, pp. 219-269. 